\title{
Pathogenic alpha-synuclein aggregates preferentially bind to mitochondria and affect cellular respiration
}

\author{
Xinhe Wang ${ }^{1}$, Katelyn Becker ${ }^{1}$, Nathan Levine ${ }^{1}$, Michelle Zhang ${ }^{1}$, Andrew P. Lieberman², Darren J. Moore ${ }^{1}$ and \\ Jiyan $\mathrm{Ma}^{1^{*}}$ (D)
}

\begin{abstract}
Misfolded alpha-synuclein (aSyn) is a major constituent of Lewy bodies and Lewy neurites, which are pathological hallmarks of Parkinson's disease (PD). The contribution of aSyn to PD is well established, but the detailed mechanism remains obscure. Using a model in which aSyn aggregation in primary neurons was seeded by exogenously added, preformed aSyn amyloid fibrils (PFF), we found that a majority of pathogenic aSyn (indicated by serine 129 phosphorylated aSyn, ps-aSyn) was membrane-bound and associated with mitochondria. In contrast, only a minuscule amount of physiological aSyn was mitochondrial bound. In vitro, aSyn PFF displayed a stronger binding to purified mitochondria than did aSyn monomer, revealing a preferential mitochondria binding by aggregated aSyn. This selective mitochondrial ps-aSyn accumulation was confirmed in other neuronal and animal aSyn aggregation models that do not require exogenously added PFF and, more importantly, in postmortem brain tissues of patients suffering from PD and other neurodegenerative diseases with aSyn aggregation (asynucleinopathies). We also showed that the mitochondrial ps-aSyn accumulation was accompanied by defects in cellular respiration in primary neurons, suggesting a link to mitochondrial dysfunction. Together, our results show that, contrary to physiological aSyn, pathogenic aSyn aggregates preferentially bind to mitochondria, indicating mitochondrial dysfunction as the common downstream mechanism for a-synucleinopathies. Our findings suggest a plausible model explaining the formation and the peculiar morphology of Lewy body and reveal that disrupting the interaction between ps-aSyn and the mitochondria is a therapeutic target for a-synucleinopathies.
\end{abstract}

Keywords: a-Synuclein, Aggregation, PFF, Mitochondria, Lewy body, Parkinson's disease, a-Synucleinopathies

\section{Introduction}

Parkinson's disease (PD) is a common neurodegenerative disorder in the elderly and its characteristic motor symptoms result from the loss of dopaminergic neurons in the substantia nigra [11, 37]. Mitochondrial dysfunction is sufficient to cause nigral dopaminergic neuron loss [11], which is consistent with the idea that these neurons are selectively vulnerable to various toxic insults [49]. Besides mitochondria dysfunction, genetic studies have established a causative role of dominant mutations or multiplication

\footnotetext{
* Correspondence: Jiyan.Ma@vai.org

${ }^{1}$ Center for Neurodegenerative Science, Van Andel Research Institute, 333

Bostwick Avenue N.E, Grand Rapids, MI 49503, USA

Full list of author information is available at the end of the article
}

of SNCA ( $\alpha$ Syn gene) in familial PD [8, 23, 38, 45] and the association of SNCA polymorphism with a higher risk of idiopathic PD [4], demonstrating a key contribution of $\alpha$ Syn to PD pathogenesis. $\alpha$ Syn is an intrinsically disordered protein and is abundantly expressed in neurons $[18,23]$. During the disease, $\alpha$ Syn is subjected to a variety of post-translational modifications [2]. Among them, the phosphorylation at serine 129 is most relevant to the pathogenic process and is commonly used to indicate the pathogenic $\alpha$ Syn species $[1,14]$.

In addition to $\mathrm{PD}, \alpha$ Syn aggregation is also a common pathological hallmark for a group of neurodegenerative diseases known as $\alpha$-synucleinopathies, which include PD, dementia with Lewy bodies (DLB), and multiple 
system atrophy (MSA) [15, 17]. A large body of evidence supports that the aggregation process of $\alpha$ Syn, including both oligomerization and amyloid fibril growth, is closely related to the pathogenesis of $\alpha$-synucleinopathies [23]. Notably, both increased $\alpha$ Syn expression by $S N C A$ multiplication $[8,45]$ and the presence of disease-associate $\alpha$ Syn mutations can increase the aggregation propensity of $\alpha \mathrm{Syn}$ [10]. Reducing $\alpha$ Syn clearance is also able to increase the amount of $\alpha$ Syn and thereby enhances $\alpha$ Syn aggregation and neurotoxicity [33]. Moreover, inoculating preformed $\alpha$ Syn amyloid fibrils (PFF) into wild-type mice induces endogenous $\alpha$ Syn aggregation and subsequent nigral dopaminergic neuron degeneration [29], demonstrating that $\alpha$ Syn aggregation is sufficient to cause neurodegeneration. Despite these advances, very little is known about the detailed mechanism of how $\alpha$ Syn aggregation causes neurotoxicity and contributes to the pathogenic process.

It has been reported that $\alpha$ Syn may play a role in the physiology and/or pathology of mitochondrial function [50]. Even though $\alpha$ Syn does not have a mitochondrial targeting sequence, several groups reported its localization in mitochondria or mitochondria-associated membranes (MAMs) [9, 12, 16, 25-27, 44, 47], and showed that $\alpha$ Syn affects a variety of mitochondrial functions, from $\mathrm{Ca}^{2+}$ signaling, to complex I activity, to mitochondrial morphology and dynamics [50]. However, given the well-established presynaptic localization of $\alpha$ Syn and its role in synaptic vesicle release $[5-7,18,19,21]$, it remains unclear how much physiological $\alpha$ Syn is mitochondria-associated and to what extent it affects mitochondrial function. Moreover, whether the mitochondrial $\alpha$ Syn localization contributes to the disease process and how it is related to the other major pathogenic event, $\alpha$ Syn aggregation, are completely unknown.

Some recent studies started to explore the potential relationship between pathogenic $\alpha$ Syn species and mitochondria. Using a proximity ligation assay (PLA), Di Maio et al. showed that $\alpha$ Syn oligomer and S129E phosphomimic mutant each bind TOM20 on the mitochondrial outer membrane and impair mitochondrial protein import [13]. Using exogenously added $\alpha$ Syn oligomer, Ludtman et al. showed mitochondrial localization of $\alpha$ Syn oligomer by PLA and the impairment of mitochondrial function [28]. They also showed that increased endogenous $\alpha$ Syn aggregates in neurons derived from an SNCA triplication patient were also in the vicinity of mitochondrial ATP synthase, indicating a mitochondrial localization [28]. Using human pluripotent stem cells expressing mutant $S N C A$, Ryan et al. reported fragmented mitochondria and the clustering of $\alpha$ Syn aggregates in the mitochondria of SNCA mutant neurons, but not in the isogenic control neurons [42]. The methodologies used in above studies, including transient transfection, exogenously added $\alpha$ Syn oligomers, SNCA mutant cells, and heavy dependence on imaging-based analyses, make it difficult to conclude how much pathogenic $\alpha$ Syn is associated with mitochondria; whether mitochondrial association is a main pathogenic pathway for $\alpha$ Syn aggregates; whether the mitochondrial ps- $\alpha$ Syn accumulation occurs in neurons that express wild-type $\alpha$ Syn at endogenous level, and more importantly, whether such accumulation occurs in $\alpha$-synucleinopathy patients.

To understand the cellular mechanism of $\alpha$ Syn aggregation and the resulting neurotoxicity, we adopted a highly reproducible primary neuron model developed by Volpicelli-Daley et al., in which the neuronal accumulation of ps- $\alpha$ Syn was induced by exogenously added PFF [52]. We took an unbiased approach to study the subcellular localization of $\mathrm{ps}-\alpha \mathrm{Syn}$ and found that the majority of ps- $\alpha$ Syn was associated with mitochondria. This finding was verified in other neuronal and mouse models and was also confirmed with postmortem brain tissues from $\alpha$-synucleinopathy patients. Consistent with these findings, our in vitro results showed a preferential binding of mitochondria by aggregated $\alpha$ Syn. Moreover, we have also showed that the mitochondrial accumulation of ps- $\alpha$ Syn is associated with mitochondrial respiration defects, suggesting mitochondrial dysfunction as a downstream consequence of aggregated $\alpha$ Syn.

\section{Materials and methods}

Purification of recombinant aSyn and preparation of aSyn PFF

Mouse $\alpha$ Syn was purified as previously described $[3,51]$. Purified $\alpha$ Syn was dialyzed against PBS buffer (VWR\#97062-732), aliquoted, and stored at $-80^{\circ} \mathrm{C}$. When needed, aliquots of $\alpha$ Syn were thawed, concentrated to $350 \mu \mathrm{M}$, and shaken at $37^{\circ} \mathrm{C}$ for $7 \mathrm{~d}$ at $1000 \mathrm{rpm}$ to prepare mature mouse $\alpha$ Syn preformed fibrils (PFF) [51]. PFF was aliquoted and stored at $-80^{\circ} \mathrm{C}$. All PFF preparations were verified by the thioflavin $\mathrm{T}$ fluorescence assay and by imaging using a Tecnai G2 Spirit TWIN transmission electron microscope. The purification of human $\alpha$ Syn and the preparation of hPFF were performed with the same procedures described above.

\section{Primary neuronal culture}

P1 mouse or rat cortical neurons were isolated and cultured essentially according to a previously published protocol [51]. At $7 \mathrm{~d}$ in vitro (DIV), neurons were treated with or without $140 \mathrm{nM} \alpha$ Syn monomer or PFF. Treated neurons were cultured as usual and collected after 8-9 d or after a specified time. PFF was sonicated before each usage with a water-bath cup-horn sonicator (Misonix XL2020) at 50\% power for $5 \mathrm{~min}$. For proteasomal inhibition, primary neurons were treated with 
DMSO or $100 \mathrm{nM}$ epoxomicin for $8 \mathrm{~h}$, and after incubation, cell lysates or homogenates were prepared.

\section{Immunofluorescence staining and proximity ligation assay (PLA)}

Neurons at 16 DIV were fixed with $4 \%$ formaldehyde in culture medium at $37^{\circ} \mathrm{C}$ for $15 \mathrm{~min}$ and then permeabilized with $0.2 \%$ Triton-100 for $10 \mathrm{~min}$ at room temperature. PLA staining was performed with antiTOM20 (Abcam, 1:100) and anti- $\alpha$ Syn phospho (Ser129) (Abcam, 1:5000) using Duolink In Situ Detection Reagents (Sigma\# DUO92008), following the protocol provided by the manufacturer. For immunofluorescence staining, antiMAP2 (Sigma, 1:250), anti- $\alpha$ Syn phospho (Ser129)(Abcam, 1:1000), and anti-TOM20 (Abcam 1:100) were used as primary antibodies. Alexa 488-conjugated goat anti-mouse IgG and Alexa 594-conjugated goat antirabbit IgG (ThermoFisher) were used as secondary antibodies. Images were visualized with an Olympus IX83 microscope and a Nikon A1plus-RSi scanning confocal microscope. The Pearson's correlation coefficient was calculated with Nikon Elements Analysis AR5.11.00 (Nikon). For the proteinase $\mathrm{K}$ digestion experiment in Fig. 1h, fixed neurons were incubated

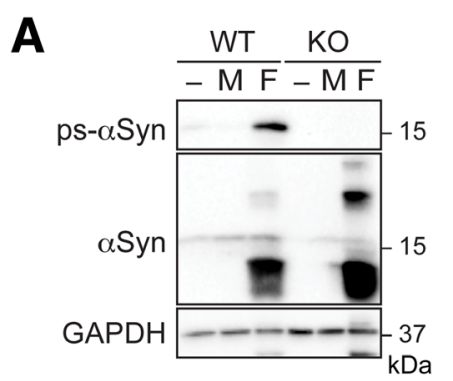

\section{B}

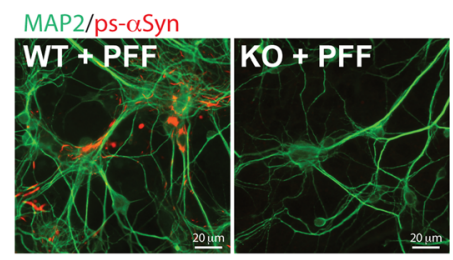

C

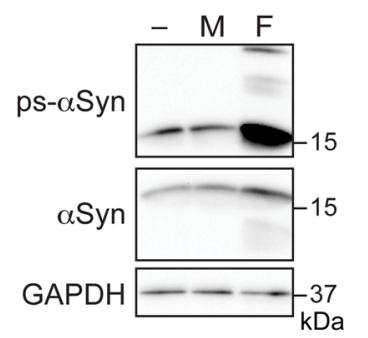

$\mathbf{E}$

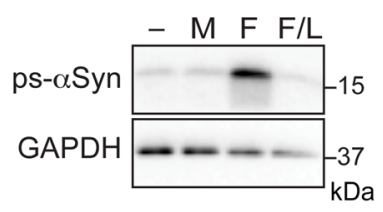

D

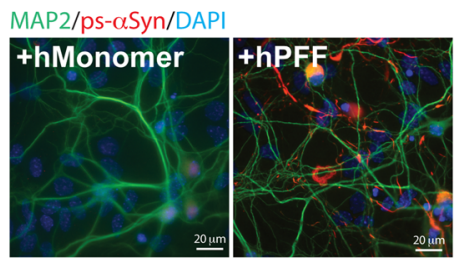

$\mathbf{F}$

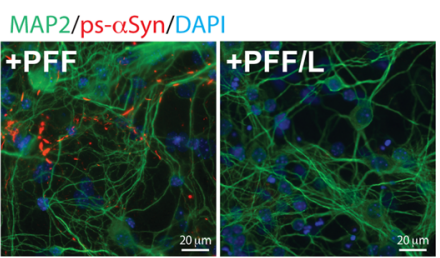

H

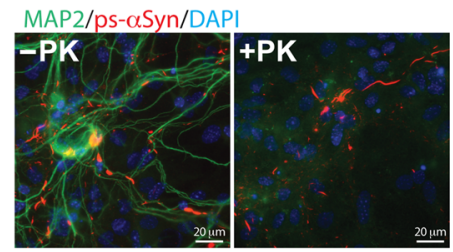

Fig. 1 PFF treatment induces ps-aSyn in primary neurons. a, b Primary cortical neurons derived from wild-type (WT) and aSyn knockout (KO) mice were untreated (-) or treated with aSyn monomer (M) or PFF (F) as indicated. $\mathbf{c}$, d Primary cortical neurons derived from OVX mice were untreated (-) or treated with human aSyn monomer (M) or PFF (F). e, f Primary neurons derived from wild-type mice were untreated (-) or treated with aSyn monomer (M), aSyn PFF (F), or lysozyme PFF (F/L). ps-aSyn (in a, $\mathbf{c}$, and e) and total aSyn (in a and $\mathbf{c}$ ) were detected by immunoblot analysis and GAPDH was used as a loading control. In b, d, and f, ps-aSyn was visualized by immunofluorescence staining (red), and MAP2 stain (green) was used as a neuronal marker. g Primary neurons, untreated (-) or treated with aSyn monomer (M) or PFF (F), were sequentially extracted with TBS, 1\% Triton/TBS, and 2\% SDS/TBS. The presence of ps-aSyn and total aSyn was detected by immunoblot analysis. $\mathbf{h}$ PFF-treated neurons were incubated with or without a $10-\mathrm{min} 10 \mu \mathrm{g} / \mathrm{mL}$ PK digestion at $37^{\circ} \mathrm{C}$ prior to immunofluorescence staining to detect psaSyn (red) and MAP2 (green) 
with $10 \mu \mathrm{g} / \mathrm{mL}$ proteinase $\mathrm{K}$ at $37^{\circ} \mathrm{C}$ for 10 min before the immunofluorescence staining.

\section{Sequential extraction}

At DIV 16, neurons were homogenized into TBS buffer (50 mM Tris, $150 \mathrm{mM} \mathrm{NaCl}, \mathrm{pH} 7.5$ ), sonicated at $50 \%$ power for $5 \mathrm{~min}$ (Misonix XL2020), and centrifuged at $100,000 \times g$ for $30 \mathrm{~min}$ at $4{ }^{\circ} \mathrm{C}$. The pellets were resuspended in 1\% Triton-100 in TBS, sonicated, and centrifuged as described above. The supernatant was collected as the Triton-soluble fraction. The pellet was resuspended in $2 \%$ SDS in TBS.

\section{Differential centrifugation to separate mitochondrial and cytosolic/microsomal fractions}

Primary cortical neurons were washed once with icecold neuronal membrane buffer (NMB, $250 \mathrm{mM}$ sucrose, $20 \mathrm{mM}$ HEPES, $10 \mathrm{mM} \mathrm{KCl}, 1.5 \mathrm{mM} \mathrm{MgCl}_{2}, 1 \mathrm{mM}$ EGTA, $1 \mathrm{mM}$ EDTA, and $1 \mathrm{mM}$ DTT, pH 7.5) and then were homogenized in NMB plus proteinase and phosphatase inhibitors $(\mathrm{NMB}+)$. Dissected spinal cords of transgenic mice, or brain tissue from patients were directly homogenized in $\mathrm{NMB}+$. The homogenates were centrifuged at $600 \times g$ (for cells) or $700 \times g$ (for tissue) at $4{ }^{\circ} \mathrm{C}$ for $5 \mathrm{~min}$ to remove nuclei and unbroken cells. The supernatants were used as the postnuclear supernatant (PNS). To separate mitochondrial and cytosolic/microsomal fractions, the PNS was centrifuged at $10,000 \times g$ for $5 \mathrm{~min}$ at $4{ }^{\circ} \mathrm{C}$. The supernatants were collected as cytosolic/microsomal fractions, and the pellets (the mitochondrial fraction) were resuspended in $\mathrm{NMB}+$.

\section{Density gradient}

For the iodixanol gradient analysis, either PNS or isolated mitochondria were mixed with iodixanol stock solution to reach the final concentration of $36 \%$. The mixture $(0.8 \mathrm{~mL})$ was loaded to the bottom of the ultracentrifuge tube. On top of it, $0.8 \mathrm{~mL}$ of $31 \%$ and $0.8 \mathrm{~mL}$ of $10 \%$ iodixanol were sequentially laid. After centrifugation at $52,000 \times g$ for $4 \mathrm{~h}$ at $4{ }^{\circ} \mathrm{C}$ (ThermoFisher rotor S55-S), $0.2 \mathrm{~mL}$ fractions were collected from top to bottom. Discontinuous sucrose gradient analysis was performed as previously described [53].

\section{Western blots}

Samples were separated on 16\% SDS polyacrylamide gel or pre-made $4-20 \%$ gradient SDS polyacrylamide gel (ThermoFisher) and transferred to PVDF membranes (GE Healthcare). The membranes were fixed with $4 \%$ paraformaldehyde and $0.1 \%$ glutaraldehyde for $30 \mathrm{~min}$ at room temperature and probed with various antibodies. The primary antibodies were anti- $\alpha$ Syn phospho (Ser129) (Abcam 51,253, 1:2500), anti- $\alpha$ Syn (BD Biosciences clone 42, 1:2500), anti-ATPIF1 (ThermoFisher,
1:1500), anti-ATP5A (Abcam, 1:1000), anti-GAPDH (Stressgen, 1:1000), anti-Calnexin (Sressgen, 1:1000), and anti-Syntaxin 6 (Sigma, 1:1000). The HRP-conjugated goat anti-mouse or anti-rabbit IgG antibodies (Bio-Rad) were used as the secondary antibodies. Signals were developed by ECL 2 substrate (Pierce) and scanned with ChemiDoc (Bio-Rad). The signal intensity was measured with the FIJI software.

\section{Mitochondrial stress test}

Primary cortical neurons were seeded in Agilent Seahorse 96-well plate at $1.0 \times 10^{4}$ per well. At DIV 7, neurons were treated with either $\alpha$ Syn monomer or PFF. At DIV 14, the oxygen consumption rate was measured with Seahorse $\mathrm{XF}^{\mathrm{e}} 96$ (Agilent) after sequentially adding $1 \mu \mathrm{M}$ oligomycin, $1.25 \mu \mathrm{M}$ FCCP (carbonyl cyanide-p-trifluoromethoxyphenylhydrazone), and $0.5 \mu \mathrm{M}$ rotenone/antimycin A (XF Cell Mito Stress Test Kit from Agilent).

\section{Animals}

C57BL/6J mice were from Van Andel Institute internal colony. CD (Sprague Dawley) IGS rats were obtained from Charles River. Transgenic mice expressing human A53T $\alpha$ Syn from the mouse prion protein promoter (line G2-3, kindly provided by Dr. Michael Lee, University of Minnesota) were described previously [24]. The bilateral intramuscular PFF injection was performed according to a previously published protocol [43] with slight modification. Briefly, the PFF used for the intramuscular injection was prepared by shaking $350 \mu \mathrm{M} \alpha$ Syn at $1000 \mathrm{rpm}$ for $2 \mathrm{~d}$ at $37^{\circ} \mathrm{C}$. PFF was aliquoted and stored at $-80^{\circ} \mathrm{C}$. Before each injection, PFF was diluted to $0.1 \mu \mathrm{g} / \mu \mathrm{L}$ and sonicated with the water-bath cup-horn sonicator (Misonix XL2020) at $25 \%$ power for $2 \mathrm{~h}$, and then $10 \mu \mathrm{L}$ of PFF $(0.1 \mu \mathrm{g} / \mu \mathrm{l})$ or $10 \mu \mathrm{L}$ PBS was injected into biceps femoris on each hindlimb.

\section{Statistical analysis}

Statistical analyses were performed with GraphPad Prism software version 6.05.

\section{Results}

PFF-induced ps-aSyn in primary neurons

We first characterized the PFF-induced synucleinopathy model in primary neurons. Our results showed that adding PFF (Additional file 1: Figure S1) was sufficient to induce the phosphorylation of endogenous $\alpha$ Syn and no ps- $\alpha$ Syn was detected in PFF-treated primary neurons derived from $\alpha$ Syn knockout mice (Fig. 1a). Immunofluorescence staining verified that $\mathrm{ps}-\alpha \mathrm{Syn}$ was accumulated in the neurons labeled with the neuronal marker MAP2 (Fig. 1b). Using an antibody detecting total $\alpha$ Syn, we found that the majority of $\alpha$ Syn in PFF-treated 
wild-type or $\alpha$ Syn knockout neurons was cleaved (Fig. 1a). Because the only $\alpha$ Syn in the knockout neurons was from PFF, this result suggested that the majority of the exogenously added PFF was truncated in neurons. The induction of ps- $\alpha$ Syn was specific to the addition of $\alpha$ Syn PFF; adding the same amount of $\alpha$ Syn monomer did not produce ps- $\alpha$ Syn. Moreover, the exogenously added monomer was barely detectable by immunoblot analysis after 9 days (Fig. 1a, monomer in $\alpha$ Syn knockout neurons), suggesting a more rapid clearance than that of exogenously added PFF. The same result was obtained with rat primary neurons (Additional file 1: Figure S2).

To determine whether the findings can be reproduced with human $\alpha$ Syn, we cultured neurons derived from the BAC transgenic mice (OVX mice) that express human wild-type $\alpha$ Syn at twice the endogenous level of mouse $\alpha$ Syn and on a mouse $\alpha$ Syn knockout background [20]. These neurons were either untreated or treated with human $\alpha$ Syn monomer or PFF prepared with human $\alpha$ Syn (hPFF). Immunoblot analysis (Fig. 1c) revealed that ps- $\alpha$ Syn was induced in neurons treated with hPFF, but not in neurons untreated or treated with human $\alpha$ Syn monomer. Immunofluorescence staining confirmed that ps- $\alpha$ Syn was in neurons (Fig. 1d).

To ensure that the observed effects were not because of toxic effects of the amyloid fibrils, we compared $\alpha$ Syn PFF with fibrils of another amyloidogenic protein, lysozyme (Fig. 1e and f). The ps- $\alpha$ Syn was accumulated only in $\alpha$ Syn PFF-treated neurons, not in those treated with lysozyme fibrils, suggesting the ps- $\alpha$ Syn was likely resulted from seeding by $\alpha$ Syn PFF.

We also determined the aggregation status of ps- $\alpha$ Syn by sequential detergent extraction. ps- $\alpha$ Syn was enriched in Triton- and SDS-soluble fractions, whereas total $\alpha$ Syn in untreated cells appeared mainly in the TBS-soluble fraction (Fig. 1g). Immunofluorescence staining revealed that

A

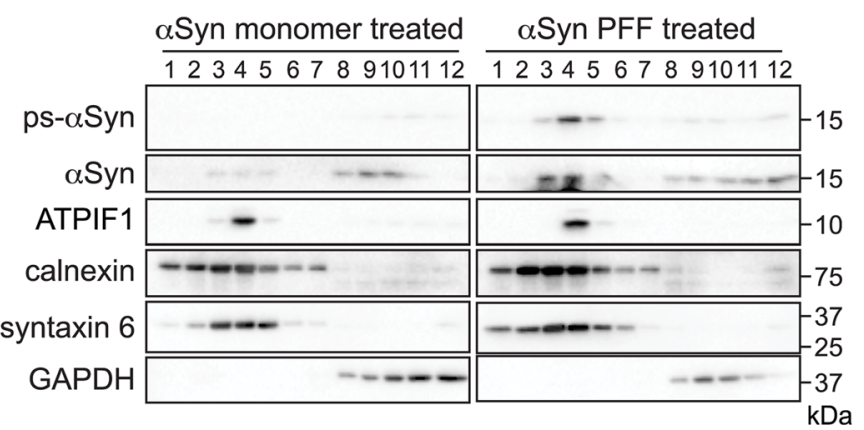

B

cyto
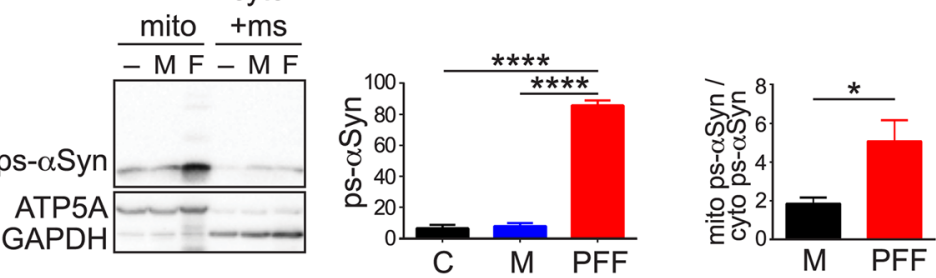

C

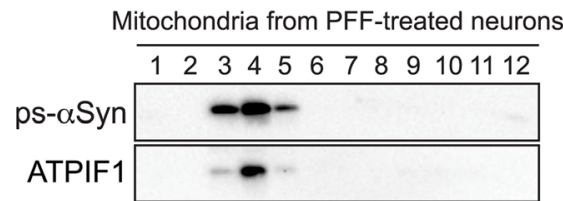

Fig. 2 Accumulation of PFF-induced ps-aSyn in mitochondria. a PNS prepared from aSyn monomer-treated or PFF-treated primary neurons were subjected to iodixanol gradient separation. The presence of each protein was detected by immunoblot analyses. b PNS from untreated (-) or aSyn monomer (M)- or PFF (F)-treated rat primary neurons were subjected to differential centrifugation to separate the mitochondrial and cytosolic/microsomal fractions, which were verified by immunoblot analyses with antibodies against mitochondrial ATP synthase (ATP5A) and cytosolic GAPDH, respectively. The ps-aSyn in these fractions was detected by immunoblot analysis. The bar graph in the middle shows the average \pm standard error of mitochondrial ps-aSyn from five independent experiments. " $C$ " represents control. Statistical significance was determined by one-way ANOVA followed by Dunnett's multiple comparisons test $(F=322.8 ; n=5 ; p<0.0001)$. The bar graph on the right shows the ratio of ps-aSyn in the mitochondria over ps-aSyn in the cytosolic/microsomal fraction, which was the average \pm standard error of three independent experiments. Statistical significance was determined by paired $t$-test $(n=3 ; p=0.048)$. * represents $p<0.05$. c The mitochondria isolated from PFF-treated neurons were subjected to iodixanol gradient separation, and the presence of ps-aSyn and the mitochondrial marker ATPIF1 was detected by immunoblot analyses 
PFF-induced ps- $\alpha$ Syn resisted proteinase $\mathrm{K}$ digestion (Fig. 1h). These results led us to conclude that the majority of PFF-induced ps- $\alpha$ Syn in primary neurons was aggregated.

\section{Preferential binding of PFF-induced ps-aSyn to mitochondria}

To determine the subcellular localization of ps- $\alpha$ Syn, postnuclear supernatant (PNS) was prepared from neurons treated with $\alpha$ Syn monomer or PFF and subjected to iodixanol density-gradient separation (Fig. 2a). The majority of total $\alpha$ Syn in monomer-treated neurons remained at the bottom, co-migrating with the cytosolic marker GAPDH (glyceraldehyde 3-phosphate dehydrogenase). The ps- $\alpha$ Syn in PFF-treated neurons, however, migrated to the upper, membrane-bound fractions. Among membrane markers, ps- $\alpha$ Syn appeared to be concentrated in
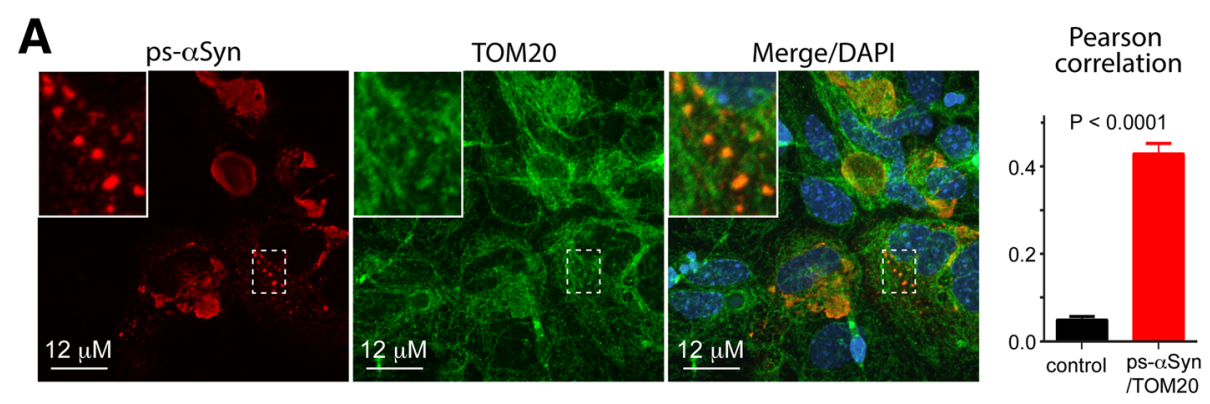

B
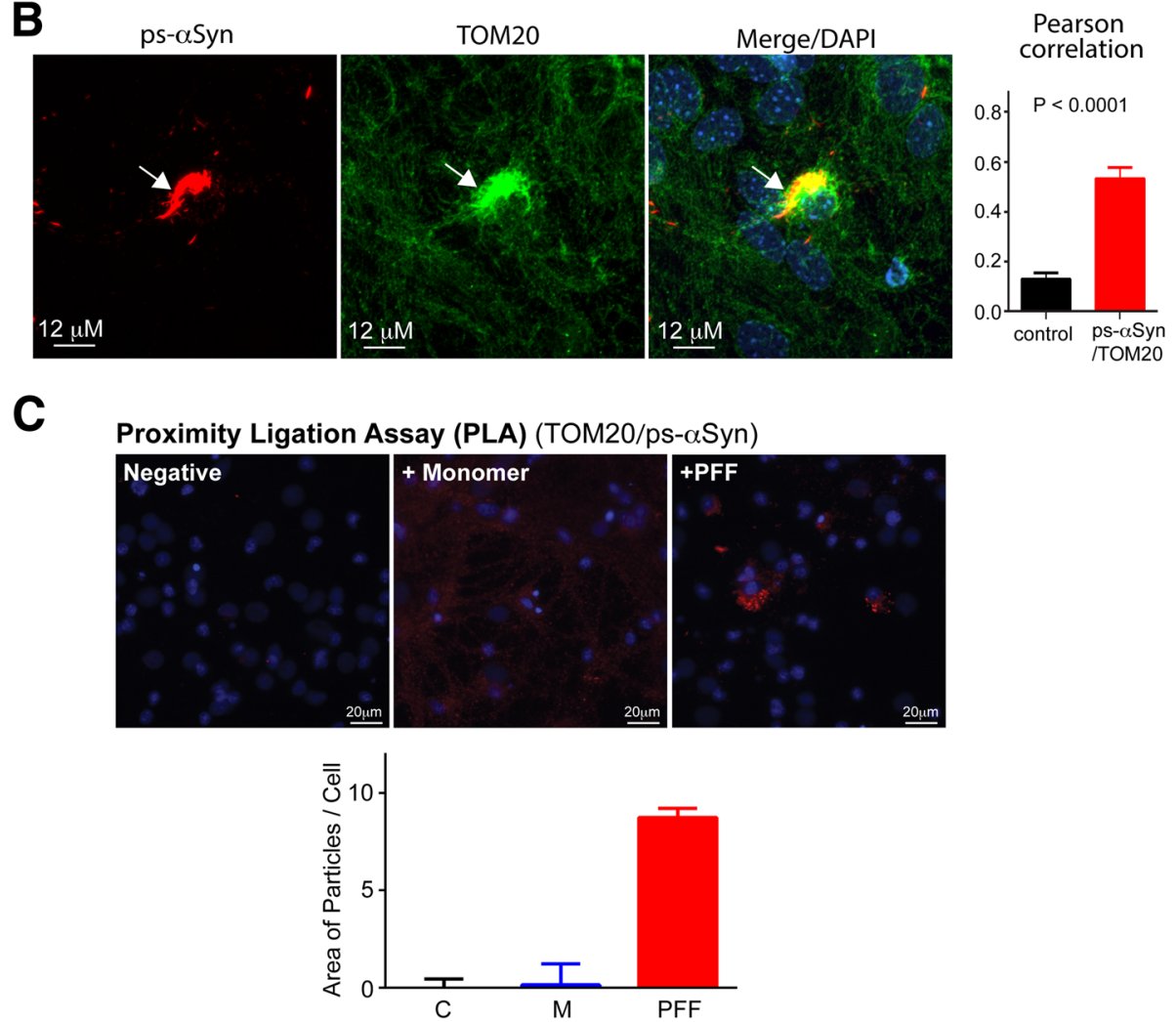

Fig. 3 Co-localization of PFF-induced ps-aSyn with mitochondria marker TOM20. a Primary cortical neurons were prepared from OVX mice, cultured for 7 days, then treated with hPFF (PFF prepared with human aSyn), cultured for another $9 \mathrm{~d}$, and subjected to immunofluorescence staining with antibodies against ps-aSyn and TOM20. Inserts are enlarged images of the areas indicated by the dashed boxes. $\mathbf{b}$ Mouse primary neurons were treated with PFF and subjected to immunofluorescence staining with antibodies against ps-aSyn and TOM20. Arrows point to the area with strong ps-aSyn accumulation, but weak TOM20 staining. The Pearson correlation coefficient in $\mathbf{a}$ and $\mathbf{b}$ was determined from 12 individual cells. Statistic difference was determined by an unpaired t-test $(n=12 ; p<0.0001)$. c A proximity ligation assay (PLA) with antibodies against ps-aSyn and TOM20 was performed in primary neurons treated with aSyn monomer or PFF. Representative images include left, negative control (omitting anti-TOM20 antibody); center, aSyn monomer-treated neurons; right, PFF-treated neurons. The bar graph shows average \pm standard error of three independent experiments with six areas quantified per experiment. Statistical analysis was performed with one-way ANOVA followed by Dunnett's multiple comparisons test $(F=66.40 ; n=6 ; p<0.0001)$ 
the peak fraction of mitochondrial marker ATPIF1 (Fig. 2a), which is similar to the finding using a discontinuous sucrose density gradient (Additional file 1: Figure S3) [53]. These results led us to hypothesize that the PFF-induced ps- $\alpha$ Syn may be mitochondria-bound.

We tested this possibility by separating the cell homogenates into mitochondrial and cytosolic/microsomal fractions by differential centrifugation. We found that majority of ps- $\alpha$ Syn was indeed in the mitochondrial fraction (Fig. 2b and Additional file 1: Figure S4). To rule out the possibility that the appearance of ps- $\alpha$ Syn in mitochondria fraction was simply due to its aggregation status, we subjected isolated mitochondria to a density gradient that was optimized for mitochondria purification. The ps- $\alpha$ Syn co-migrated with mitochondrial marker ATPIF1 to the top of the gradient (Fig. 2c), confirming its association with the membranous mitochondria.

Immunofluorescence staining was used to determine the subcellular localization of PFF-induced ps- $\alpha$ Syn. Compared with ps- $\alpha$ Syn in PFF-treated mouse primary neurons (Fig. $3 b$ ), the human ps- $\alpha$ Syn in hPFF-treated OVX neurons appeared to be less clumpy or less tightly packed (Fig. 3a), which presumably reflects the high propensity of mouse $\alpha$ Syn to aggregate [41]. Nevertheless, co-localization of ps- $\alpha$ Syn with the mitochondrial outer membrane protein TOM20 was detected in both hPFF-treated OVX neurons (Fig. 3a) and in PFF-treated mouse neurons (Fig. 3b). In mouse neurons, we consistently observed a reduced TOM20 staining in areas having strong ps- $\alpha$ Syn staining (Fig. 3b, arrows), which was also observed in PFF-treated rat neurons
(Additional file 1: Figure S5, arrows). We reasoned that the observation is likely due to the high aggregation propensity of mouse $\alpha$ Syn, which forms tightly packed aggregates and interferes with the detection of mitochondrial markers by immunofluorescence staining. To verify the co-localization of ps- $\alpha$ Syn and TOM20, we performed a proximity ligation assay (PLA) with antibodies against ps- $\alpha$ Syn and TOM20 in PFF-treated rat primary neurons. We found that positive signals were only detected in primary neurons treated with PFF (Fig. 3c), which supported our conclusion that the majority of PFF-induced ps- $\alpha$ Syn is associated with mitochondria.

\section{Aggregated aSyn, but not physiological aSyn, preferentially binds to mitochondria}

To compare the amount of physiological $\alpha$ Syn monomer and pathogenic $\alpha$ Syn aggregates associated with mitochondria, we isolated mitochondria from neurons treated with $\alpha$ Syn monomer or PFF and then subjected them to a density gradient. As expected, mitochondria migrated to the upper fraction (Fig. 4a, top panel). Using mitochondria isolated from neurons treated with $\alpha$ Syn monomer, we found little $\alpha$ Syn associated with the mitochondria (Fig. 4a, second panel). However, in PFF-treated neurons, a significant amount of $\alpha$ Syn (both full-length and truncated) was in the mitochondrial fraction, which co-migrated with ps- $\alpha$ Syn (Fig. 4a, third and fourth panels). Some of the truncated $\alpha$ Syn might derive from exogenously added PFF because PFF was efficiently cleaved in these neurons (Fig. 1a). Nevertheless, this result

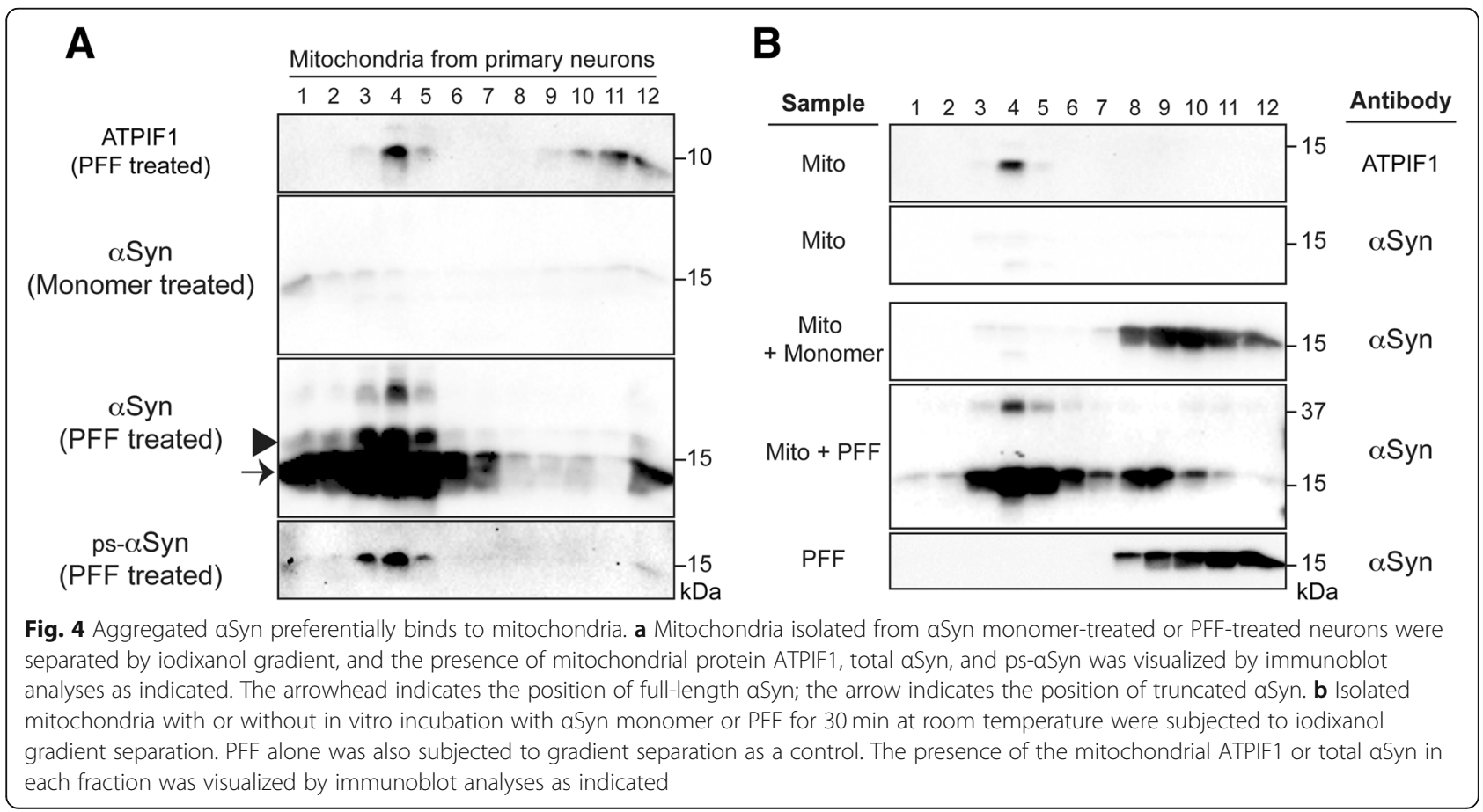


suggested to us that both full-length and truncated $\alpha$ Syn aggregates can be associated with mitochondria and that ps- $\alpha$ Syn only reflects a portion of full-length $\alpha$ Syn.

The large amount of $\alpha$ Syn accumulated in the mitochondrial fraction of PFF-treated neurons suggested to us that aggregated $\alpha$ Syn may have a preference to bind to mitochondria. To test this possibility, we purified mitochondria from untreated primary neurons, incubated isolated mitochondria with $\alpha$ Syn monomer or PFF in vitro, and then subjected the mixture to the density-gradient analysis. The purified mitochondria contained very little endogenous $\alpha$ Syn (Fig. 4 b, second panel), which is consistent with the idea that very little physiological $\alpha$ Syn binds to mitochondria under normal conditions. After incubation with $\alpha$ Syn monomer or PFF, the majority of $\alpha$ Syn monomer remained at the bottom, but the majority of $\alpha$ Syn PFF co-migrated with the mitochondrial protein ATPIF1 to the upper fractions (Fig. 4b, third and fourth panels). As a control, $\alpha$ Syn PFF alone (i.e. without incubation with mitochondria) was also subject to the gradient separation, and it remained at the bottom (Fig. 4b, bottom panel). Therefore, our results suggested that relative to $\alpha$ Syn monomer, aggregated $\alpha$ Syn preferentially binds to the mitochondria.
Preferential binding of ps-aSyn to mitochondria in other neuronal and transgenic mouse models

The mitochondrial accumulation of ps- $\alpha$ Syn in PFF-treated neurons could be a result of the preferential mitochondrial binding by PFFs, which subsequently seed the conversion of endogenous $\alpha$ Syn on the surface of mitochondria. To rule out this possibility, we tested other neuronal and transgenic mouse models that do not require exogenously added PFF. First, we treated primary neurons with epoxomycin, a proteasome inhibitor, which is able to induce the accumulation of ps- $\alpha$ Syn (Fig. 5a) [40]. When the PNS prepared from epoxomycin-treated neurons were separated into mitochondrial and cytosolic/microsomal fractions, the ps- $\alpha$ Syn was enriched in the mitochondrial fraction (Fig. 5b). Second, we used a transgenic mouse model overexpressing human $\alpha$ Syn carrying the pathogenic A53T mutation [24]. This mouse model develops spontaneous neurodegeneration at old age with major pathological changes in the spinal cord and brain stem [30], and the disease can be significantly accelerated by intramuscular PFF inoculation [43]. Immunoblot analysis revealed a huge amount of ps- $\alpha$ Syn accumulated in the spinal cord of diseased mice, which was not present in young control mice that received PBS inoculation (Fig. 5c). Notably, regardless whether the disease occurred spontaneously or accelerated

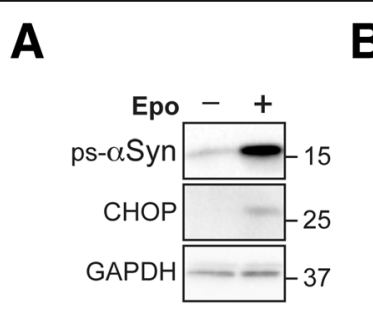

B
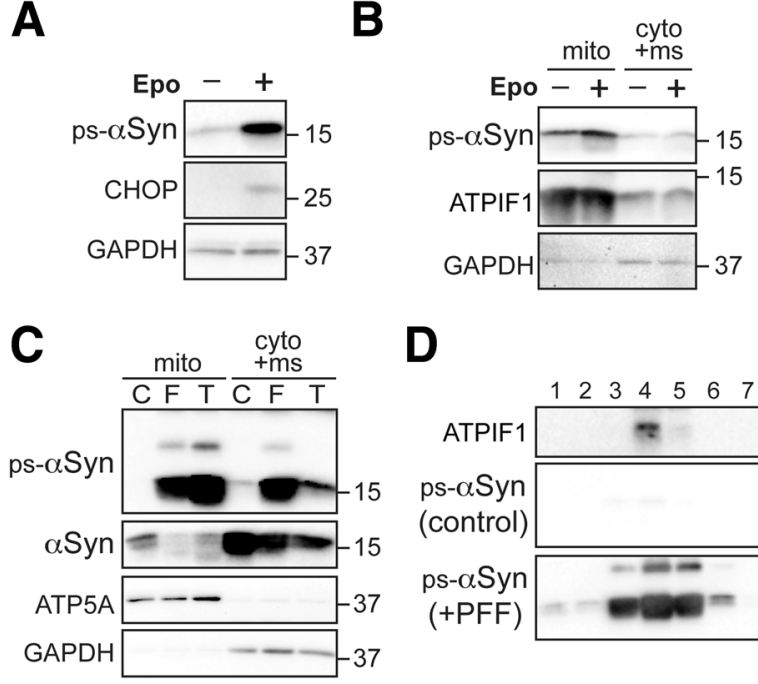

D

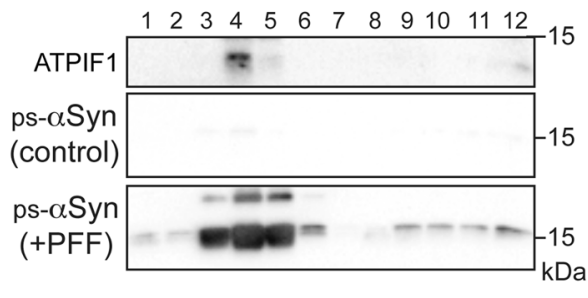

Fig. 5 Association of ps-aSyn with mitochondria in primary neuron and transgenic mouse models. a ps-aSyn and CCAAT-enhancer-binding protein homologous protein (CHOP) accumulations in primary neurons treated with the proteasomal inhibitor epoxomicin (Epo) were determined by immunoblot analyses. $\mathbf{b}$ The homogenates prepared from Epo-treated (+) or untreated (-) neurons were separated into mitochondrial fraction (mito) and cytosolic/microsomal fraction (cyto+ms) by differential centrifugation. The mitochondrial enrichment of proteasomal-inhibitioninduced ps-aSyn, mitochondrial ATPIF1, and the cytosolic marker GAPDH were detected by immunoblot analyses. c An A53T transgenic mouse was sacrificed at terminal stage of neurodegeneration (T, $305 \mathrm{~d}$ of age); Littermates of A53T transgenic mice intramuscularly injected with PBS (C) or aSyn PFF (F) were sacrificed when the PFF-injected mouse developed terminal neurodegeneration at $67 \mathrm{~d}$ after injection (135 $\mathrm{d}$ of age). Spinal cord homogenates prepared from these mice were separated into mitochondrial (mito) and cytosolic/microsomal (cyto+ms) fractions by differential centrifugation. ps-aSyn, total aSyn, mitochondrial ATP synthase (ATP5A), and cytosolic GAPDH were detected by immunoblot analyses. $\mathbf{d}$ Mitochondria isolated from the spinal cords of transgenic mice that received PBS (control) or aSyn PFF (+PFF) injections were subjected to iodixanol gradient separation, and the presence of ps-aSyn and ATPIF1 was detected by immunoblot analyses 


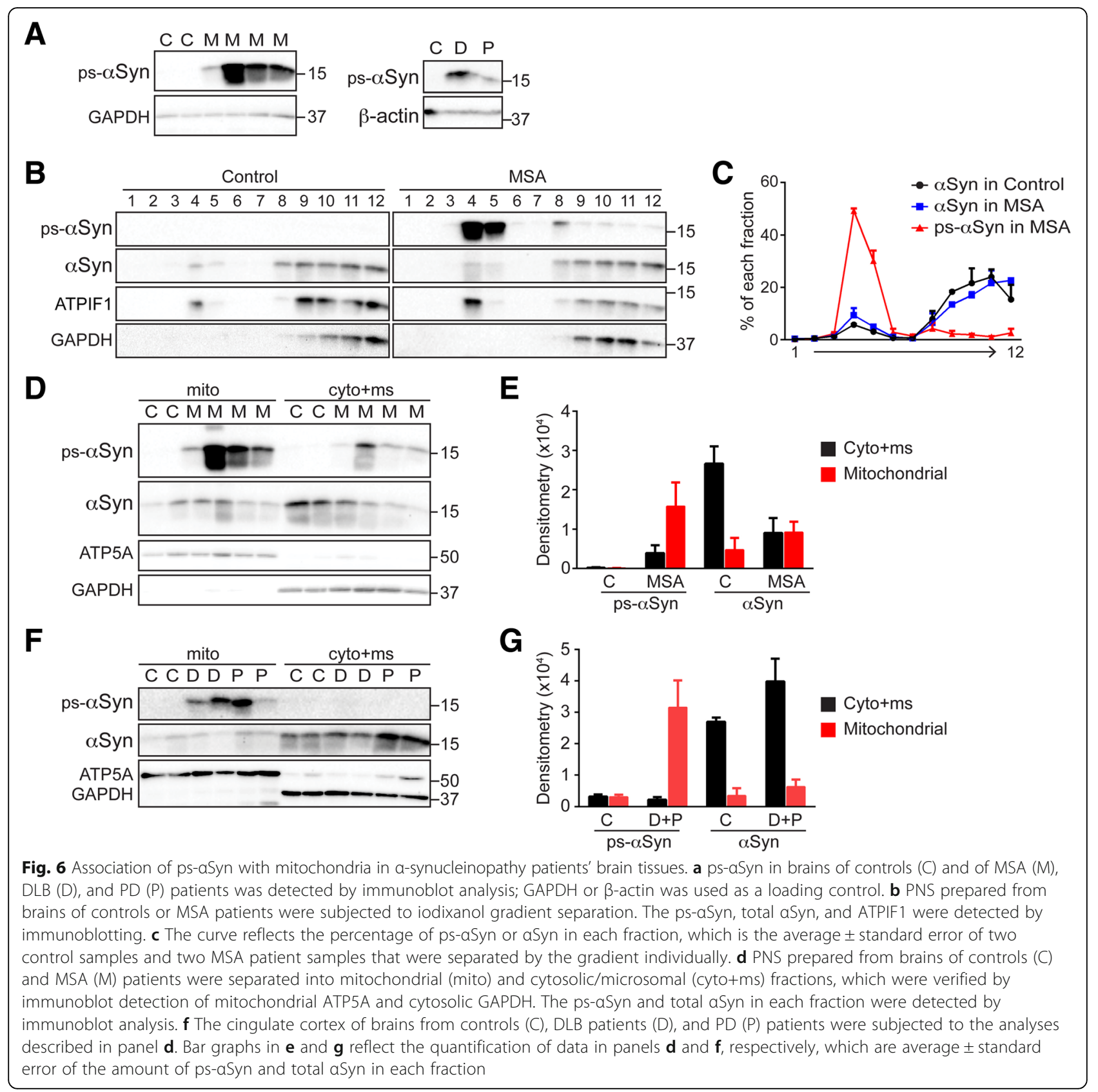

by PFF-inoculation, the ps- $\alpha$ Syn was mainly found in the mitochondrial fraction (Fig. 5c). In contrast, the majority of total $\alpha$ Syn, including the endogenous mouse $\alpha$ Syn and the $\alpha$ Syn from the human A53T $\alpha$ Syn transgene, was in the cytosolic/microsomal fraction (Fig. 5c). To rule out the possibility that the result was influenced by the aggregation status of ps- $\alpha$ Syn, we subjected the mitochondria fraction to the density-gradient analysis. The majority of ps- $\alpha$ Syn co-migrated with the mitochondrial marker ATPIF1 to upper fractions (Fig. 5d), confirming that ps- $\alpha$ Syn was indeed associated with mitochondria. Therefore, we concluded that the mitochondrial ps- $\alpha$ Syn accumulation is not due to exogenously added PFF, but is an inherent property of $\alpha$ Syn aggregates.

The majority of ps-aSyn was associated with mitochondria in postmortem brain tissues from asynucleinopathy patients

To determine whether ps- $\alpha$ Syn is associated with mitochondria in patients' brains, we tested postmortem patient brain tissues from various $\alpha$-synucleinopathies (Additional file 1: Table S1). As expected, ps- $\alpha$ Syn was detected in the postmortem tissues from such patients, but not in tissue from controls (Fig. 6a). When PNS prepared from the 


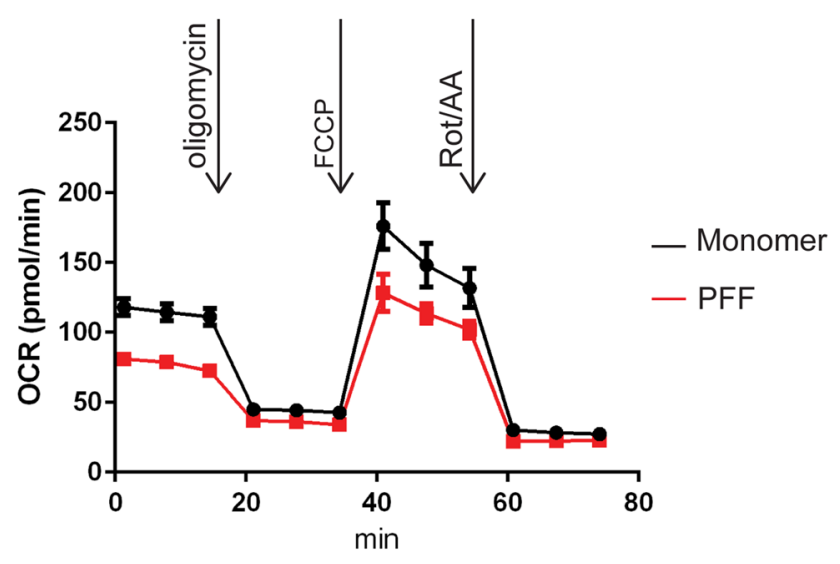

Basal OCR (pmol/min)

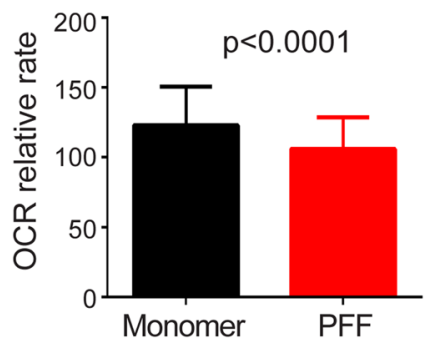

\section{Maximal OCR (pmol/min)}

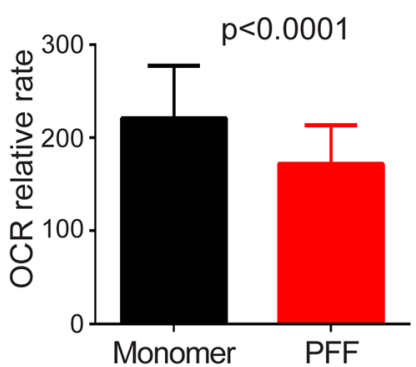

Fig. 7 PFF treatment of primary neurons causes respiration defects. Mouse cortical neurons treated with aSyn monomer or PFF were subjected to the mitochondrial stress test measuring oxygen consumption rate. The differences in the basal and maximal respiration levels are shown in the bar graphs, which represent the average \pm standard error of three independent experiments with 15-20 wells of cells per experiment. Statistical significance was determined by two-way ANOVA (for basal level, $F=33.66 ; \mathrm{p}<0.0001$; for maximal level, $\mathrm{F}=48.13 ; P<0.0001$ )

striatum of MSA patient brains was subjected to the density-gradient separation, the majority of total $\alpha$ Syn remained at the bottom, similar to that of controls (Fig. 6b). The mitochondrial marker ATPIF1 migrated to the upper fractions, but a portion of it remained in the bottom, which could be due to the disintegration of some mitochondria in postmortem tissue. Remarkably, the majority of ps- $\alpha$ Syn migrated to upper fractions of the gradient and co-migrated with ATPIF1, which is completely different from total $\alpha$ Syn (Fig. $6 b$ and c). When the homogenates from MSA patients were subjected to fractionation by differential centrifugation, the preferential enrichment of ps- $\alpha$ Syn in the mitochondrial fraction was obvious (Fig. $6 \mathrm{~d}$ and e). Similar results were also observed with homogenates prepared from the cingulate cortex of PD and DLB patients (Fig. $6 \mathrm{f}$ and g). Together, these results suggest to us that in patients suffering from $\alpha$-synucleinopathies, ps- $\alpha$ Syn was also preferentially associated with the mitochondria.

\section{Mitochondrial ps-aSyn accumulation is associated with defects in cellular respiration}

To determine whether the ps- $\alpha$ Syn accumulation in the mitochondria affects mitochondrial function, we subjected the primary neurons treated with $\alpha$ Syn monomer or PFF to the mitochondrial stress test. The PFF-treated neurons consistently had a higher number of trifluoromethoxy carbonylcyanide phenylhydrazone (FCCP)-unresponsive wells (Additional file 1: Figure S6), indicating a weaker tolerance to FCCP treatment. More importantly, both the basal and maximum respiration capacities were significantly lower in PFF-treated neurons (Fig. 7), indicating that mitochondrial ps- $\alpha$ Syn accumulation does affect mitochondrial function.

\section{Discussion}

Our results revealed a preferential accumulation of ps- $\alpha$ Syn in the mitochondria, connecting two major pathogenic events of $\alpha$-synucleinopathies, i.e., $\alpha$ Syn misfolding and mitochondrial dysfunction. The fact that ps- $\alpha$ Syn from three different $\alpha$-synucleinopathies was associated with mitochondria suggests to us that this is a general property of $\alpha$ Syn aggregates and is independent of the variable conformations of misfolded $\alpha$ Syn in different diseases [34]. Moreover, our results also showed that the majority of physiological $\alpha$ Syn is not associated with mitochondria, which is consistent with previous studies $[5-7,18,19,21]$ and suggests that the 


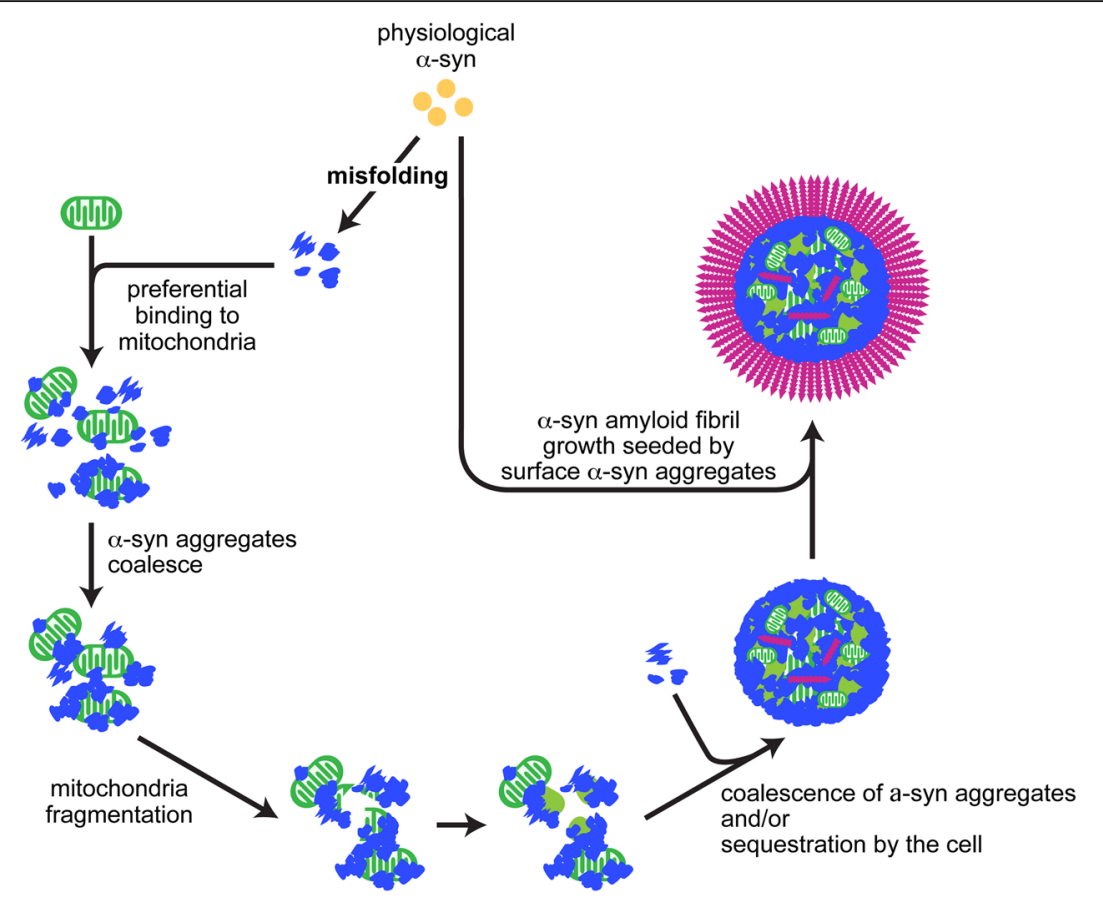

$\alpha$-synuclein

13-synuclein aggregates

$\alpha$-synuclein
amyloid fibril

(IJ) mitochondria

vesicular structures

derived from fragmented mitochondrial membranes

Fig. 8 A schematic model of Lewy body formation

mitochondrial ps- $\alpha$ Syn accumulation is most likely not due to the phosphorylation of mitochondria-associated physiological $\alpha$ Syn. Instead, it is likely resulted from the inherent preference of pathogenic $\alpha$ Syn aggregates to bind to mitochondria, which is consistent with findings from our in vitro mitochondria binding study (Fig. 4b).

Our study also revealed that the mitochondrial ps- $\alpha$ Syn accumulation is accompanied by defects in mitochondrial activity. Together with the selective vulnerability of mitochondria in the energy-demanding nigral dopaminergic neurons $[36,48,49]$, our findings may explain the fact that certain $\alpha$ Syn mutations or $S N C A$ duplication cause disease similar to idiopathic PD [8] and that polymorphism of $\alpha$ Syn gene could be a risk factor for idiopathic PD [4]. Because the preferential binding by $\alpha$ Syn aggregates is not limited to mitochondria from nigral dopaminergic neurons, the ps- $\alpha$ Syn mitochondrial connection may also contribute to the degeneration of other neuronal types, which may lead to the cognitive and behavioral disturbances in DLB or PDD (Parkinson's disease dementia) patients and in patients carrying SNCA triplication [32, 35, 45]. This conclusion is consistent with a recent report of fragmented mitochondria and clustering of $\alpha$ Syn aggregates in the mitochondria of neurons differentiated from human iPSCs carrying $\alpha$ Syn mutations [42] and the report of mitochondrial dysfunction in neurons differentiated from iPSCs derived from a patient carrying $\alpha$ Syn triplication [28].
Our findings also support the faithfulness of changes in PFF-induced models $[29,52]$ to the pathogenesis of $\alpha$-synucleinopathies. The study of postmortem human tissues reflects the end stage of diseases, but the PFF-induced model allows us to study the dynamic cellular consequences of $\alpha$ Syn aggregation. Notably, some ps- $\alpha$ Syn did appear in the TBS-soluble fraction, and a large portion of it appeared in the Triton-soluble fraction (Fig. 1g), which presumably represents various states of polymerization, from a soluble oligomeric state to an amyloid fibril state. Because the majority of ps- $\alpha$ Syn co-migrated with mitochondria (Fig. 2b and c), it is reasonable to predict that not only $\alpha$ Syn aggregates in the fibril state, but also some of the $\alpha$ Syn oligomers, bind to mitochondria as well.

Because of the intrinsic tendency of $\alpha$ Syn aggregates to coalesce [23], binding of $\alpha$ Syn aggregates to mitochondria is not the end, but rather the middle, of a dynamic process (Fig. 8). The mitochondria-bound $\alpha$ Syn aggregates may further coalesce and/or the protein aggregate-bound mitochondria may be subjected to cellular clearance system. Thus, it is plausible to postulate that mitochondrial dysfunction could result from the accumulation of $\alpha$ Syn aggregates on or in mitochondria, which may interact with mitochondrial proteins and interfere with their normal functions. Alternatively, mitochondrial dysfunction may result from the tendency to coalesce by mitochondria-bound $\alpha$ Syn aggregates, which will disturb 
the normal mitochondria movement, dynamics, and morphology. When $\alpha$ Syn aggregation becomes overwhelming, it could also disrupt mitochondria, resulting in fragments of mitochondrial membranes (Fig. 8). This process, together with cell's ability to concentrate misfolded proteins [22], may ultimately lead to the formation of Lewy Bodies.

It is important to note that the mitochondria-associated $\alpha$ Syn aggregates remain competent to seed $\alpha$ Syn monomer to form ordered aggregates. While $\alpha$ Syn aggregates in the middle of Lewy bodies are either already coalesced without reactive surface or crowded by membranous structures without the access to $\alpha$ Syn monomer, the $\alpha$ Syn aggregates on the surface remain competent to seed and have the accessibility to $\alpha$ Syn monomer (Fig. 8). Thus, in neurons where $\alpha$ Syn monomer is available, $\alpha$ Syn amyloid fibrils can be grown on the surface of Lewy bodies, resulting in a ring of densely packed $\alpha$ Syn fibrils that reacts strongly with antibody during immunohistochemical staining [31]. Therefore, our model predicts that at the end stage, Lewy bodies or Lewy neurites may contain membranous structures, mitochondria, $\alpha$ Syn aggregates in complex with mitochondria and vesicles, and $\alpha \mathrm{Syn}$ fibrils $[31,39,46]$.

\section{Conclusion}

Our study provides solid evidence supporting the preferential binding of pathogenic $\alpha$ Syn species to mitochondria; suggests mitochondrial dysfunction as the common downstream pathogenic mechanism for $\alpha$ Syn aggregation; and points out that disrupting the binding of pathogenic $\alpha$ Syn species to mitochondria could be beneficial to patients suffering from $\alpha$-synucleinopathies.

\section{Additional file}

Additional file 1: Table S1. Postmortem tissues. Figure S1. Electron microscopic images of aSyn PFF before and after sonication. Figure S2. PFF-induced ps-aSyn in rat neurons. Primary cortical neurons were untreated $(-)$ or treated with aSyn monomer (M) or PFF (F) as indicated. The presence of ps-aSyn was detected by immunofluorescence staining (red), and MAP2 stain (green) was used as a neuronal marker. Nuclei were stained with DAPI. The lower left panel shows a representative immunoblot of ps-aSyn and total aSyn; immunoblot analysis of GAPDH was performed to verify equal loading. The bar graph represents the average \pm standard error of five independent experiments. Statistical significance was determined by one-way ANOVA followed by a Dunnett's multiple comparison test ( $\mathrm{F}=51.21, n=5, p<0.0001)$. Figure S3. Postnuclear supernatant (PNS) prepared from PFF-treated primary neurons was separated using a discontinuous sucrose gradient, and fractions were collected from the top to the bottom. The presence of ps-aSyn, mitochondrial ATPIF1, calnexin (endoplasmic reticulum), and syntaxin 6 (Golgi) in each fraction were detected by immunoblot analysis. Figure S4. PFF-induced ps-aSyn accumulated in the mitochondria of mouse primary neurons. PNS of mouse primary neurons treated with either aSyn monomer (M) or PFF (F) were separated into mitochondrial (mito) and cytosolic/microsomal (cyto+ms) fractions. The presence of ps-aSyn, mitochondrial ATP synthase (ATP5A), and cytosolic GAPDH was detected by immunoblot analysis. Figure S5. Immunofluorescence staining was performed on PFF-treated rat primary neurons with antibodies against ps-aSyn and TOM20 as indicated. Arrows indicate cellular areas with strong ps-aSyn stain, but weak TOM20 stain. The top and bottom panels are two separated images. Figure S6. PFF-treated neurons were less responsive to FCCP treatment. The graph represents the average \pm standard error of four independent experiments. The statistic difference was determined by paired $t$-test $(p=0.0029 ; n=4)$. (DOCX $1025 \mathrm{~kb}$ )

\section{Acknowledgements}

We would like to thank Dr. Corinne Esquibel at optical imaging core of Van Andel Institute for providing technical support for image acquisition and analysis; David Nadziejka at Van Andel Institute for technical editing the manuscript. We also would like to thank the staff at Michigan brain bank for their support.

\section{Funding}

This work is supported in part by NIH grants R21NS101676, R01NS060729, and P30AG053760.

\section{Availability of data and materials}

All data generated in this study are presented in this article and its online supplementary materials.

\section{Authors' contributions}

JM conceived and designed experiments, analyzed data, and wrote the manuscript. XW designed, planned, and performed experiments, analyzed data, contributed to the writing of the manuscript. KB expressed and purified recombinant aSyn, prepared and validated aSyn PFF used in cell culture experiments. NL contributed to the setup of primary neuronal culture and the rat primary neuron culture. $M Z$ performed part of the immunoblot analyses. APL and DJM provided critical reagents for the study and analyzed the data. All authors discussed the results and commented on the manuscript. All authors read and approved the final manuscript.

Ethics approval and consent to participate

This study was conducted in compliance with federal and institutional guidelines. All experiments were approved by appropriate committees at the Van Andel Research Institute.

\section{Consent for publication}

Not applicable.

Competing interests

The authors declare that they have no competing interests.

\section{Publisher's Note}

Springer Nature remains neutral with regard to jurisdictional claims in published maps and institutional affiliations.

\section{Author details}

${ }^{1}$ Center for Neurodegenerative Science, Van Andel Research Institute, 333 Bostwick Avenue N.E, Grand Rapids, MI 49503, USA. ²Department of Pathology, University of Michigan Medical School, Ann Arbor MI48109, USA.

Received: 8 March 2019 Accepted: 10 March 2019

Published online: 14 March 2019

\section{References}

1. Anderson JP, Walker DE, Goldstein JM, de Laat R, Banducci K, Caccavello RJ, Barbour R, Huang J, Kling K, Lee M, Diep L, Keim PS, Shen X, Chataway T, Schlossmacher MG, Seubert P, Schenk D, Sinha S, Gai WP, Chilcote TJ (2006) Phosphorylation of Ser-129 is the dominant pathological modification of alpha-synuclein in familial and sporadic Lewy body disease. J Biol Chem 281:29739-29752. https://doi.org/10.1074/jbc.M600933200

2. Barrett PJ, Timothy Greenamyre J (2015) Post-translational modification of alpha-synuclein in Parkinson's disease. Brain Res 1628:247-253. https://doi. org/10.1016/j.brainres.2015.06.002

3. Becker K, Wang X, Vander Stel K, Chu Y, Kordower J, Ma J (2018) Detecting alpha Synuclein seeding activity in formaldehyde-fixed MSA patient tissue by PMCA. Mol Neurobiol 55:8728-8737. https://doi.org/10. 1007/s12035-018-1007-y 
4. Billingsley KJ, Bandres-Ciga S, Saez-Atienzar S, Singleton AB (2018) Genetic risk factors in Parkinson's disease. Cell Tissue Res 373:9-20. https://doi.org/ 10.1007/s00441-018-2817-y

5. Burre J, Sharma M, Sudhof TC (2012) Systematic mutagenesis of alphasynuclein reveals distinct sequence requirements for physiological and pathological activities. J Neurosci 32:15227-15242. https://doi.org/10.1523/ JNEUROSCI.3545-12.2012

6. Burre J, Sharma M, Sudhof TC (2014) Alpha-Synuclein assembles into higher-order multimers upon membrane binding to promote SNARE complex formation. Proc Natl Acad Sci U S A 111:E4274-E4283. https://doi. org/10.1073/pnas.1416598111

7. Burre J, Sharma M, Tsetsenis T, Buchman V, Etherton MR, Sudhof TC (2010) Alpha-synuclein promotes SNARE-complex assembly in vivo and in vitro. Science 329:1663-1667. https://doi.org/10.1126/science.1195227

8. Chartier-Harlin MC, Kachergus J, Roumier C, Mouroux V, Douay X, Lincoln S, Levecque C, Larvor L, Andrieux J, Hulihan M, Waucquier N, Defebvre L, Amouyel P, Farrer M, Destee A (2004) Alpha-synuclein locus duplication as a cause of familial Parkinson's disease. Lancet 364:1167-1169. https:/doi.org/ 10.1016/S0140-6736(04)17103-1

9. Chinta SJ, Mallajosyula JK, Rane A, Andersen JK (2010) Mitochondrial alphasynuclein accumulation impairs complex I function in dopaminergic neurons and results in increased mitophagy in vivo. Neurosci Lett 486:235239. https://doi.org/10.1016/j.neulet.2010.09.061

10. Conway KA, Harper JD, Lansbury PT (1998) Accelerated in vitro fibril formation by a mutant alpha-synuclein linked to early-onset Parkinson disease. Nat Med 4:1318-1320. https://doi.org/10.1038/3311

11. Dauer W, Przedborski S (2003) Parkinson's disease: mechanisms and models. Neuron 39:889-909

12. Devi L, Raghavendran V, Prabhu BM, Avadhani NG, Anandatheerthavarada HK (2008) Mitochondrial import and accumulation of alpha-synuclein impair complex I in human dopaminergic neuronal cultures and Parkinson disease brain. J Biol Chem 283:9089-9100. https://doi.org/10.1074/jbc.M710012200

13. Di Maio R, Barrett PJ, Hoffman EK, Barrett CW, Zharikov A, Borah A, Hu X, McCoy J, Chu CT, Burton EA, Hastings TG, Greenamyre JT (2016) Alpha-Synuclein binds to TOM20 and inhibits mitochondrial protein import in Parkinson's disease. Sci Transl Med 8:342ra378. https:/doi.org/10.1126/scitranslmed.aaf3634

14. Fujiwara H, Hasegawa M, Dohmae N, Kawashima A, Masliah E, Goldberg MS, Shen J, Takio K, Iwatsubo T (2002) Alpha-Synuclein is phosphorylated in synucleinopathy lesions. Nat Cell Biol 4:160-164. https://doi.org/10.1038/ncb748

15. Goedert M, Jakes R, Spillantini MG (2017) The Synucleinopathies: twenty years on. J Park Dis 7:S53-S71. https://doi.org/10.3233/JPD-179005

16. Guardia-Laguarta C, Area-Gomez E, Rub C, Liu Y, Magrane J, Becker D, Voos W, Schon EA, Przedborski S (2014) Alpha-Synuclein is localized to mitochondria-associated ER membranes. J Neurosci 34:249-259. https://doi. org/10.1523/JNEUROSCI.2507-13.2014

17. Halliday GM, Holton JL, Revesz T, Dickson DW (2011) Neuropathology underlying clinical variability in patients with synucleinopathies. Acta Neuropathol 122:187-204. https://doi.org/10.1007/s00401-011-0852-9

18. Iwai A, Masliah E, Yoshimoto M, Ge N, Flanagan L, de Silva HA, Kittel A, Saitoh T (1995) The precursor protein of non-a beta component of Alzheimer's disease amyloid is a presynaptic protein of the central nervous system. Neuron 14:467-475

19. Jakes R, Spillantini MG, Goedert M (1994) Identification of two distinct synucleins from human brain. FEBS Lett 345:27-32

20. Janezic S, Threlfell S, Dodson PD, Dowie MJ, Taylor TN, Potgieter D, Parkkinen L, Senior SL, Anwar S, Ryan B, Deltheil T, Kosillo P, Cioroch M, Wagner K, Ansorge O, Bannerman DM, Bolam JP, Magill PJ, Cragg SJ, WadeMartins R (2013) Deficits in dopaminergic transmission precede neuron loss and dysfunction in a new Parkinson model. Proc Natl Acad Sci U S A 110: E4016-E4025. https://doi.org/10.1073/pnas.1309143110

21. Kahle PJ, Neumann M, Ozmen L, Muller V, Jacobsen H, Schindzielorz A, Okochi M, Leimer U, van Der Putten H, Probst A, Kremmer E, Kretzschmar HA, Haass C (2000) Subcellular localization of wild-type and Parkinson's disease-associated mutant alpha -synuclein in human and transgenic mouse brain. J Neurosci 20:6365-6373

22. Kopito RR (2000) Aggresomes, inclusion bodies and protein aggregation. Trends Cell Biol 10:524-530

23. Lashuel HA, Overk CR, Oueslati A, Masliah E (2013) The many faces of alphasynuclein: from structure and toxicity to therapeutic target. Nat Rev Neurosci 14:38-48. https://doi.org/10.1038/nrn3406
24. Lee MK, Stirling W, Xu Y, Xu X, Qui D, Mandir AS, Dawson TM, Copeland NG, Jenkins NA, Price DL (2002) Human alpha-synuclein-harboring familial Parkinson's disease-linked ala-53 --> Thr mutation causes neurodegenerative disease with alpha-synuclein aggregation in transgenic mice. Proc Natl Acad Sci U S A 99:8968-8973. https://doi.org/10.1073/pnas.132197599

25. Li WW, Yang R, Guo JC, Ren HM, Zha XL, Cheng JS, Cai DF (2007) Localization of alpha-synuclein to mitochondria within midbrain of mice. Neuroreport 18:1543-1546. https://doi.org/10.1097/WNR.0b013e3282f03db4

26. Liu G, Zhang C, Yin J, Li X, Cheng F, Li Y, Yang H, Ueda K, Chan P, Yu S (2009) Alpha-Synuclein is differentially expressed in mitochondria from different rat brain regions and dose-dependently down-regulates complex I activity. Neurosci Lett 454:187-192. https://doi.org/10.1016/j.neulet.2009.02.056

27. Ludtmann MH, Angelova PR, Ninkina NN, Gandhi S, Buchman VL, Abramov AY (2016) Monomeric alpha-Synuclein exerts a physiological role on brain ATP synthase. J Neurosci 36:10510-10521. https://doi.org/10.1523/ JNEUROSCI.1659-16.2016

28. Ludtmann MHR, Angelova PR, Horrocks MH, Choi ML, Rodrigues M, Baev AY, Berezhnov AV, Yao Z, Little D, Banushi B, Al-Menhali AS, Ranasinghe RT, Whiten DR, Yapom R, Dolt KS, Devine MJ, Gissen P, Kunath T, Jaganjac M, Pavlov EV, Klenerman D, Abramov AY, Gandhi S (2018) Alpha-synuclein oligomers interact with ATP synthase and open the permeability transition pore in Parkinson's disease. Nat Commun 9:2293. https://doi.org/10.1038/ s41467-018-04422-2

29. Luk KC, Kehm V, Carroll J, Zhang B, O'Brien P, Trojanowski JQ, Lee VM (2012) Pathological alpha-synuclein transmission initiates Parkinson-like neurodegeneration in nontransgenic mice. Science 338:949-953. https://doi. org/10.1126/science.1227157

30. Martin LJ, Pan Y, Price AC, Sterling W, Copeland NG, Jenkins NA, Price DL, Lee MK (2006) Parkinson's disease alpha-synuclein transgenic mice develop neuronal mitochondrial degeneration and cell death. J Neurosci 26:41-50. https://doi.org/10.1523/JNEUROSCI.4308-05.2006

31. Olanow CW, Perl DP, DeMartino GN, McNaught KS (2004) Lewy-body formation is an aggresome-related process: a hypothesis. Lancet Neurol 3: 496-503. https://doi.org/10.1016/S1474-4422(04)00827-0

32. Olgiati S, Thomas A, Quadri M, Breedveld GJ, Graafland J, Eussen H, Douben H, de Klein A, Onofrj M, Bonifati V (2015) Early-onset parkinsonism caused by alpha-synuclein gene triplication: clinical and genetic findings in a novel family. Parkinsonism Relat Disord 21:981-986. https://doi.org/10.1016/j. parkreldis.2015.06.005

33. Pan T, Kondo S, Le W, Jankovic J (2008) The role of autophagy-lysosome pathway in neurodegeneration associated with Parkinson's disease. Brain 131:1969-1978. https://doi.org/10.1093/brain/awm318

34. Peng C, Gathagan RJ, Covell DJ, Medellin C, Stieber A, Robinson JL, Zhang B, Pitkin RM, Olufemi MF, Luk KC, Trojanowski JQ, Lee VM (2018) Cellular milieu imparts distinct pathological alpha-synuclein strains in alphasynucleinopathies. Nature 557:558-563. https://doi.org/10.1038/s41586-0180104-4

35. Peng C, Gathagan RJ, Lee VM (2018) Distinct alpha-Synuclein strains and implications for heterogeneity among alpha-Synucleinopathies. Neurobiol Dis 109:209-218. https://doi.org/10.1016/j.nbd.2017.07.018

36. Pissadaki EK, Bolam JP (2013) The energy cost of action potential propagation in dopamine neurons: clues to susceptibility in Parkinson's disease. Front Comput Neurosci 7:13. https://doi.org/10.3389/fncom.2013.00013

37. Poewe W, Seppi K, Tanner CM, Halliday GM, Brundin P, Volkmann J, Schrag $A E$, Lang AE (2017) Parkinson disease. Nature reviews Disease primers 3: 17013. https://doi.org/10.1038/nrdp.2017.13

38. Polymeropoulos MH, Lavedan C, Leroy E, Ide SE, Dehejia A, Dutra A, Pike B, Root H, Rubenstein J, Boyer R, Stenroos ES, Chandrasekharappa S, Athanassiadou A, Papapetropoulos T, Johnson WG, Lazzarini AM, Duvoisin RC, Di lorio G, Golbe LI, Nussbaum RL (1997) Mutation in the alpha-synuclein gene identified in families with Parkinson's disease. Science 276:2045-2047

39. Power JH, Barnes OL, Chegini F (2017) Lewy bodies and the mechanisms of neuronal cell death in Parkinson's disease and dementia with Lewy bodies. Brain Pathol 27:3-12. https://doi.org/10.1111/bpa.12344

40. Rideout HJ, Larsen KE, Sulzer D, Stefanis L (2001) Proteasomal inhibition leads to formation of ubiquitin/alpha-synuclein-immunoreactive inclusions in PC12 cells. J Neurochem 78:899-908

41. Rochet JC, Conway KA, Lansbury PT Jr (2000) Inhibition of fibrillization and accumulation of prefibrillar oligomers in mixtures of human and mouse alpha-synuclein. Biochemistry 39:10619-10626 
42. Ryan T, Bamm W, Stykel MG, Coackley CL, Humphries KM, JamiesonWilliams R, Ambasudhan R, Mosser DD, Lipton SA, Harauz G, Ryan SD (2018) Cardiolipin exposure on the outer mitochondrial membrane modulates alpha-synuclein. Nat Commun 9:817. https://doi.org/10.1038/s41467-01803241-9

43. Sacino AN, Brooks M, Thomas MA, McKinney AB, Lee $S$, Regenhardt RW, McGarvey NH, Ayers Jl, Notterpek L, Borchelt DR, Golde TE, Giasson BI (2014) Intramuscular injection of alpha-synuclein induces CNS alphasynuclein pathology and a rapid-onset motor phenotype in transgenic mice. Proc Natl Acad Sci U S A 111:10732-10737. https://doi.org/10. 1073/pnas.1321785111

44. Shavali S, Brown-Borg HM, Ebadi M, Porter J (2008) Mitochondrial localization of alpha-synuclein protein in alpha-synuclein overexpressing cells. Neurosci Lett 439:125-128. https://doi.org/10.1016/..neulet.2008.05.005

45. Singleton $A B$, Farrer $M$, Johnson J, Singleton $A$, Hague $S$, Kachergus J, Hulihan M, Peuralinna T, Dutra A, Nussbaum R, Lincoln S, Crawley A, Hanson M, Maraganore D, Adler C, Cookson MR, Muenter M, Baptista M, Miller D, Blancato J, Hardy J, Gwinn-Hardy K (2003) Alpha-Synuclein locus triplication causes Parkinson's disease. Science 302:841. https://doi. org/10.1126/science. 1090278

46. Spillantini MG, Crowther RA, Jakes R, Hasegawa M, Goedert M (1998) AlphaSynuclein in filamentous inclusions of Lewy bodies from Parkinson's disease and dementia with lewy bodies. Proc Natl Acad Sci U S A 95:6469-6473

47. Subramaniam SR, Vergnes L, Franich NR, Reue K, Chesselet MF (2014) Region specific mitochondrial impairment in mice with widespread overexpression of alpha-synuclein. Neurobiol Dis 70:204-213. https://doi. org/10.1016/j.nbd.2014.06.017

48. Surmeier DJ (2018) Determinants of dopaminergic neuron loss in Parkinson's disease. FEBS J 285:3657-3668. https://doi.org/10.1111/ febs.14607

49. Surmeier DJ, Obeso JA, Halliday GM (2017) Selective neuronal vulnerability in Parkinson disease. Nat Rev Neurosci 18:101-113. https://doi.org/10.1038/ nrn.2016.178

50. Vicario M, Cieri D, Brini M, Cali T (2018) The close encounter between alphaSynuclein and mitochondria. Front Neurosci 12:388. https://doi.org/10.3389/ fnins.2018.00388

51. Volpicelli-Daley LA, Luk KC, Lee VM (2014) Addition of exogenous alphasynuclein preformed fibrils to primary neuronal cultures to seed recruitment of endogenous alpha-synuclein to Lewy body and Lewy neurite-like aggregates. Nat Protoc 9:2135-2146. https://doi.org/10.1038/nprot.2014.143

52. Volpicelli-Daley LA, Luk KC, Patel TP, Tanik SA, Riddle DM, Stieber A, Meaney DF, Trojanowski JQ, Lee VM (2011) Exogenous alpha-synuclein fibrils induce Lewy body pathology leading to synaptic dysfunction and neuron death. Neuron 72:57-71. https://doi.org/10.1016/j.neuron.2011.08.033

53. Wang X, Wang F, Arterburn L, Wollmann R, Ma J (2006) The interaction between cytoplasmic prion protein and the hydrophobic lipid core of membrane correlates with neurotoxicity. J Biol Chem 281:13559-13565. https://doi.org/10.1074/jbc.M512306200

Ready to submit your research? Choose BMC and benefit from:

- fast, convenient online submission

- thorough peer review by experienced researchers in your field

- rapid publication on acceptance

- support for research data, including large and complex data types

- gold Open Access which fosters wider collaboration and increased citations

- maximum visibility for your research: over $100 \mathrm{M}$ website views per year

At BMC, research is always in progress.

Learn more biomedcentral.com/submissions 